\title{
Charged Lifshitz Black Holes
}

\author{
M. H. Dehghani \\ Physics Department and Biruni Observatory, College of Sciences, \\ Shiraz University, Shiraz 71454, Iran \\ E-mail: mhd@shirazu.ac.ir
}

\section{R. B. Mann}

Department of Physics, University of Waterloo, 200 University Avenue West, Waterloo, Ontario, Canada, N2L $3 G 1$

E-Mail: rbmann@sciborg. uwaterloo.ca

\section{R. Pourhasan}

Department of Physics, University of Waterloo, 200 University Avenue West, Waterloo, Ontario, Canada, N2L $3 G 1$

E-Mail: rpourhas@uwaterloo.ca

\begin{abstract}
We investigate modifications of the Lifshitz black hole solutions due to the presence of Maxwell charge in higher dimensions for arbitrary $z$ and any topology. We find that the behaviour of large black holes is insensitive to the topology of the solutions, whereas for small black holes significant differences emerge. We generalize a relation previously obtained for neutral Lifshitz black branes, and study more generally the thermodynamic relationship between energy, entropy, and chemical potential. We also consider the effect of Maxwell charge on the effective potential between objects in the dual theory.
\end{abstract}




\section{Contents}

1. Introduction 1

2. Field Equations in $(n+1)$-dimensions 3

3. Exact Solutions 5

4. Solutions at large $r \quad 7$

5. Near horizon expansion 8

6. Numeric Solutions in $(n+1)$-dimensions 11

6.1 Uncharged Einstein-Lifshitz Solutions 11

6.2 Charged Einstein-Lifshitz Solutions 14

$\begin{array}{ll}\text { 7. Thermal behavior } & 16\end{array}$

$\begin{array}{ll}\text { 8. Wilson loop } & 17\end{array}$

$\begin{array}{ll}\text { 9. Conserved Charge } & 19\end{array}$

10. Finite Action and the Energy Density for Einstein-Maxwell-Lifshitz $\begin{array}{ll}\text { Solutions } & 20\end{array}$

11. Concluding Remarks 24

\section{Introduction}

Since the AdS/CFT correspondence was proposed by Maldacena [1] holography has been a useful tool in studying strongly coupled field theories. Specifically, holography proposes a duality between the gravitational dynamics in an asymptotically AdS spacetime and a conformal field theory on the boundary.

In recent years the idea of holographic duality has been developed beyond high energy physics to describe strongly coupled systems in condensed matter physics, such as quantum critical systems $[4,5,6,7]$. Such systems are difficult to study using traditional methods in condensed matter based on weakly interacting quasiparticles and broken symmetry. Quantum critical points have a spacetime scale invariance 
which provides a strong kinematic connection to the some versions of AdS/CFT correspondence. This scaling symmetry is based on anisotropic scaling transformation between space and time known as Lifshitz scaling

$$
t \rightarrow \lambda^{z} t, \quad r \rightarrow \lambda^{-1} r, \quad \mathbf{x} \rightarrow \lambda \mathbf{x},
$$

where $z(\geq 1)$ is a dynamical critical exponent represents the degree of anisotropy between space and time; manifestly $z=1$ exhibits relativistic systems. This scaling property (noted previously in other contexts [2]) holographically is represented in the following form of the spacetime metric [3]:

$$
d s^{2}=\ell^{2}\left(-r^{2 z} d t^{2}+\frac{d r^{2}}{r^{2}}+r^{2} d \mathbf{x}^{2}\right)
$$

where the coordinates $\left(t, r, x^{i}\right)$ are dimensionless and the only length scale in the geometry is $\ell$. Metrics asymptotic to (1.2) can be generated as solutions to the equations of motion that follow from the action:

$$
I=\frac{1}{16 \pi} \int d^{n+1} x \sqrt{-g}\left(R-2 \Lambda-\frac{1}{4} F_{\mu \nu} F^{\mu \nu}-\frac{1}{4} H_{\mu \nu} H^{\mu \nu}-\frac{C}{2} B_{\mu} B^{\mu}\right),
$$

where $\Lambda$ is the cosmological constant, $F_{\mu \nu}=\partial_{[\mu} A_{\nu]}$ with $A_{\mu}$ representing the Maxwell gauge and $H_{\mu \nu}=\partial_{[\mu} B_{\nu]}$ is the field strength of the gauge field $B_{\mu}$ with mass $m^{2}=C$.

Recently a similar action to (1.3) but without a massless gauge field (the Maxwell field) has been introduced in four dimensions [8, 9], such that background metrics with the anisotropic scale invariance (1.2) are obtained as exact solutions. An extension of these results to 5-dimensions including the massless gauge field was carried out shortly afterward [10, 12]. The introduction of the Maxwell field introduced a new length scale, allowing the gravitational system to undergo phase transitions. Working in $(4+1)$ dimensions [10], an exact charged solution for $z=6$ was obtained, and the relationship between black hole temperature and charge was numerically computed. Exact solutions in $(4+1)$ dimensions [12], and then $(n+1)$ dimensions $[13,14]$ were subsequently obtained, where $z=2 n-2$. These models yield a holographic description of a strongly coupled quantum critical point in $(n+1)$ dimensions with asymmetric scaling that models the anomalous specific heat found at low temperature in many heavy fermion compounds [13].

While some work has been done to obtain general numerical solutions for charged Lifshitz black holes with planar topologies [10, 12, 13], the general character of such solutions for general topologies and in arbitrary dimension has not been explicated up to now. Here we investigate modifications of the Lifshitz black hole solutions due to the presence of Maxwell charge in higher dimensions for arbitrary $z$. As with their neutral counterparts, we find that the behaviour of large black holes is insensitive to the topology of the solutions, whereas for small black holes significant differences emerge. 
We also investigate the thermodynamic relationship between energy, entropy, and chemical potential, obtaining a generalization of a relation previously obtained for neutral Lifshitz black holes [15, 16, 17].

Accordingly, in Sec. 2 we introduce the field equation for the metric functions and gauge field while our method is a slightly different from what has been done in [13]. In section 3 we discuss the behaviour of solutions at large $r$ and then go on to examine near horizon expansions in section 4. Section 5 starts by comparing numerical solutions in different dimensions for uncharged black holes and follows by investigating the effect that the Maxwell charge has on their behaviour. We consider the thermal behaviour of these black holes and how charge affects their temperature in section 6 . We then go on to consider how charge modifies the potential between two particles in the dual theory by investigating corrections to the Wilson loop in 4dimensions. We compute the conserved charge and the relationship between energy, entropy, and electromagnetic potential in section 8. We close our paper with some concluding remarks.

\section{Field Equations in $(n+1)$-dimensions}

Using the variational principle the field equations that follow from the action (1.3) are:

$$
\begin{aligned}
& G_{\mu \nu}+\Lambda g_{\mu \nu}=T_{\mu \nu}, \\
& \nabla^{\mu} H_{\mu \nu}=C B_{\mu}, \\
& \partial_{[\mu} B_{\nu]}=H_{\mu \nu}, \\
& \nabla^{\mu} F_{\mu \nu}=0
\end{aligned}
$$

where the equations for the massive gauge field have been rewritten in first-order form and where

$T_{\mu \nu}=-\frac{1}{2}\left(\frac{1}{4} F_{\rho \sigma} F^{\rho \sigma} g_{\mu \nu}-F_{\mu}^{\rho} F_{\rho \nu}+\frac{1}{4} H_{\rho \sigma} H^{\rho \sigma} g_{\mu \nu}-H_{\mu}^{\rho} H_{\rho \nu}+C\left[\frac{1}{2} B_{\rho} B^{\rho} g_{\mu \nu}-B_{\mu} B_{\nu}\right]\right)$

is the energy-momentum tensor of gauge fields.

The $(n+1)$-dimensional metric preserving the basic symmetries (1.1) under consideration can be written as:

$$
d s^{2}=\ell^{2}\left(-r^{2 z} f^{2}(r) d t^{2}+\frac{g^{2}(r) d r^{2}}{r^{2}}+r^{2} d \Omega_{k}^{2}\right)
$$


where

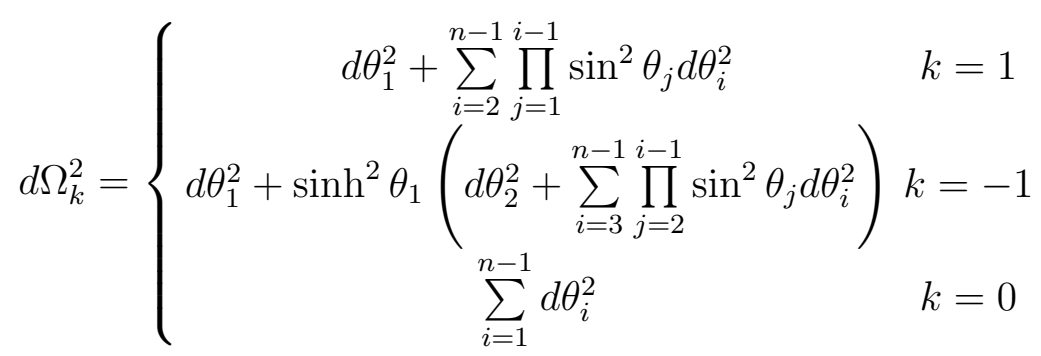

represents the metric of an $(n-1)$-dimensional hypersurface with constant curvature $(n-1)(n-2) k$ and volume $V_{n-1}$. The hypersurface is $S^{n-1}, R^{n-1}$ or $H^{n-1}$, respectively, for $k=1,0$ or -1 .

The gauge fields are assumed to be

$$
A_{t}=\ell r^{z} \kappa(r), \quad B_{t}=q \ell r^{z} f(r) j(r), \quad H_{t r}=q \ell z r^{z-1} g(r) h(r) f(r),
$$

with all other components either vanishing or being given by antisymmetrization. In order to get the asymptotic Lifshitz geometry (1.2) we demand $f(r)=g(r)=h(r)=$ $j(r)=1$ and $\kappa(r)=0$ as $r \rightarrow \infty$, which in turn imposes the following constraints

$$
\begin{aligned}
& C=\frac{(n-1) z}{\ell^{2}}, \quad q^{2}=\frac{2(z-1)}{z}, \\
& \Lambda=-\frac{(z-1)^{2}+n(z-2)+n^{2}}{2 \ell^{2}},
\end{aligned}
$$

Applying the ansatz (2.6) to the equation (2.4) yields:

$$
\left(r^{z} \kappa\right)^{\prime}=\frac{Q}{r^{n-z}} f g
$$

where $Q$ is an integration constant related to the Maxwell charge (as we will discuss later) and we have chosen boundary conditions such that the Maxwell vector potential vanishes at the horizon. Substituting (2.9) and (2.10) into eqs. (2.1-2.3), the field equations reduce to the system of first order differential equations

$$
\begin{aligned}
r \frac{d f}{d r}= & \frac{f}{4(n-1) r^{2}}\left\{2\left[(n-1)(z-1) j^{2}-z(z-1) h^{2}+(z-1)^{2}+n(z-2)+n^{2}\right] r^{2} g^{2}\right. \\
& \left.+2(n-1)\left[(n-2) k \ell^{2} g^{2}-(n+2 z-2) r^{2}\right]-Q^{2} r^{2(2-n)} g^{2}\right\}, \\
r \frac{d g}{d r}= & \frac{g}{4(n-1) r^{2}}\left\{2\left[(n-1)(z-1) j^{2}+z(z-1) h^{2}-(z-1)^{2}-n(z-2)-n^{2}\right] r^{2} g^{2}\right. \\
& \left.-2(n-1)\left[(n-2) k \ell^{2} g^{2}-n r^{2}\right]+Q^{2} r^{2(2-n)} g^{2}\right\}, \\
r \frac{d j}{d r}= & -\frac{j}{4(n-1) r^{2}}\left\{2\left[(n-1)(z-1) j^{2}-z(z-1) h^{2}+(z-1)^{2}+n(z-2)+n^{2}\right] r^{2} g^{2}\right. \\
& \left.+2(n-1)\left[(n-2) k \ell^{2} g^{2}-(n-2) r^{2}\right]-Q^{2} r^{2(2-n)} g^{2}\right\}+z g h, \\
r \frac{d h}{d r}= & (n-1)(j g-h),
\end{aligned}
$$

One can easily check that the above system will reduce to the four dimensional Einstein case introduced in $[8,9]$ if one put $n=3$ and $z=2$ and $Q=0$. 


\section{Exact Solutions}

In the previous section we introduced a set of four first order differential equations which in general can not be solved analytically because of non-linearity. However, it is possible to find some exact solutions under certain assumptions. Indeed, if one chooses the fields such that

$$
f(r)=j(r)=\frac{1}{g(r)}
$$

then the set of four ODE's reduces to three. One may easily solve the differential equation for $h(r)$ which yields:

$$
h(r)=1+\frac{\tilde{h}}{r^{n-1}}
$$

where $\tilde{h}$ is an integration constant. Now, considering the assumption (3.1) and substituting eq. (3.2), one may find that Eqs. (2.12) and (2.13) would be satisfied provided:

$$
\begin{array}{r}
2 r^{5}(2-2 n+z)\left[Q^{2}+2 z(z-1) \tilde{h}^{2}\right]+4 z r^{n+4}\left(2 z^{2}+2 n-z(n+1)-2\right) \tilde{h} \\
-4 k \ell^{2} r^{2 n+1}(n-1)(n-2)(z-2)=0 .
\end{array}
$$

There are two possibilities for solving this equation.

First, we can set $z=2(n-1)$. Then the two first terma in (3.3) are eliminated. By solving the remaining terms for $\tilde{h}$ we have

$$
\tilde{h}=\frac{(n-2)^{2} k \ell^{2} r^{n-3}}{2(n-1)(3 n-4)}
$$

Since $\tilde{h}$ should be a constant then the solutions are consistent if $k=0$ or $n=2,3$. The exact solutions for $k=0$ and arbitrary $n$ are given by [11]:

$$
h=1, \quad f^{2}=j^{2}=\frac{1}{g^{2}}=1-\frac{Q^{2}}{2(n-1)^{2} r^{2 n-2}}, \quad \kappa=\frac{Q}{(n-1) r^{n-1}}\left(1-\frac{r_{0}^{n-1}}{r^{n-1}}\right)
$$

where there is just one horizon located at

$$
r_{0}^{2}=\left[\frac{Q^{2}}{2(n-1)^{2}}\right]^{1 /(n-1)},
$$

and the metric functions start from zero at the horizon and monotonically increase to approach unity at infinity.

For $n=2$ the metric is the same for all values of $k$ and the solution is

$$
h=1, \quad f^{2}=j^{2}=\frac{1}{g^{2}}=1-\frac{Q^{2}}{2 r^{2}}, \quad \kappa=\frac{Q}{r}\left(1-\frac{r_{0}}{r}\right)
$$



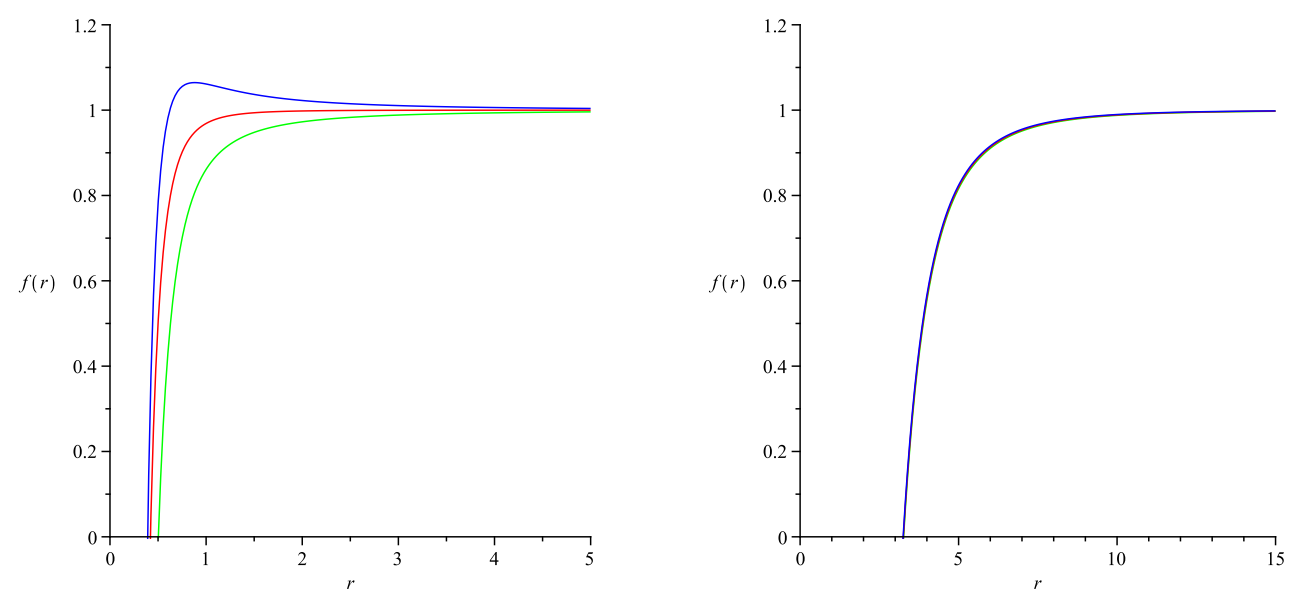

Figure 1: Metric functions $f(r)$ in 4-dimensions with: Left) $Q=0.5$ for $k=-1$ (green), $k=0$ (red) and $k=1$ (blue). Right) $Q=30$ for all $k$ 's.

while in 4 dimensions $(n=3)$ they differ for different $k$ 's [14]:

$$
h=1+\frac{k \ell^{2}}{20 r^{2}}, \quad f^{2}=j^{2}=\frac{1}{g^{2}}=1+\frac{k \ell^{2}}{10 r^{2}}-\frac{3 \ell^{4} k^{2}}{400 r^{4}}-\frac{Q^{2}}{8 r^{4}}, \quad \kappa=\frac{Q}{2 r^{2}}\left(1-\frac{r_{0}^{2}}{r^{2}}\right)
$$

One may note that in contrast to RN black holes, the four-dimensional metric functions (3.8) have just one horizon at:

$$
r_{0}^{2}=-\frac{k \ell^{2}}{20}+\frac{\sqrt{4 k^{2} \ell^{4}+50 Q^{2}}}{20}
$$

For small charges - which means small black holes - the distinction between solutions with different $k$ but the same charge are manifest. However as charge increases the black hole radius becomes larger and the distinction between metrics of different $k$ with the same charge becomes very small, as shown in Fig. (1).

The other alternative occurs for $0<z<1$. In this case the first two terms in eq. (3.3) would be canceled if one chooses:

$$
Q^{2}=2 z(1-z) \tilde{h}^{2}
$$

Now solving the remaining terms in (3.3) for $\tilde{h}$ yields:

$$
\tilde{h}=\frac{(n-1)(n-2)(2-z) k \ell^{2} r^{n-3}}{z\left(2+z-2 z^{2}\right)-z(2-z) n}
$$

which is a constant for charged solutions if $n=3$. Substituting Eqs. (3.10) and (3.11) and solving the field equations for $n=3$, the solutions are given by:

$$
\begin{aligned}
& h=1-\frac{(2-z) k \ell^{2}}{z\left(z^{2}-2 z+2\right) r^{2}}, \quad f^{2}=j^{2}=\frac{1}{g^{2}}=1+\frac{k \ell^{2}}{\left(z^{2}-2 z+2\right) r^{2}}, \\
& \kappa= \pm \sqrt{\frac{2(1-z) k^{2} \ell^{4}}{z\left(z^{2}-2 z+2\right)^{2}}} \frac{1}{r^{2}}\left(1-\frac{r_{0}^{z-2}}{r^{z-2}}\right)
\end{aligned}
$$


Therefore the 4 dimensional metric function with $0<z<1$ is a unit function for $k=0$, a naked singularity for $k=1$ and a black hole with one horizon located at $r_{0}^{2}=\ell^{2} /\left(z^{2}-2 z+2\right)$ for $k=-1$.

\section{Solutions at large $r$}

In the previous section we wrote down the field equations to higher dimensions and arbitrary $z$ (equations (2.11)-(2.14)), and here we review the general form of the asymptotic behaviour of the solutions [13].

We begin with linearizing the system in $(n+1)$-dimensions. Since we require the general metric (2.6) to asymptotically approach the Lifshitz one (1.2), we investigate the behaviour at large $r$ by applying a small perturbation for the fields

$$
\begin{aligned}
& f(r)=1+w f_{1}(r), \\
& g(r)=1+w g_{1}(r), \\
& j(r)=1+w j_{1}(r), \\
& h(r)=1+w h_{1}(r) .
\end{aligned}
$$

In the charged case we must also consider constraints on the behaviour of the gauge field to ensure Lifshitz asymptotics. We first note that the quantity $Q$ is proportional to the electric charge per unit volume, since

$$
\mathcal{Q}=\frac{1}{16 \pi \Omega_{k}} \int_{S}{ }^{*} F=\frac{1}{16 \pi \Omega_{k}} \int d \Omega_{k} r^{n-1} n^{\mu} F_{\mu \nu} u^{\nu}=\frac{1}{16 \pi \Omega_{k}} \int d \Omega_{k} r^{n-1} \frac{Q r^{1-z}}{r^{n-z}}=\frac{Q}{16 \pi}
$$

where $u^{\mu}$ and $n^{\mu}$ are the unit timelike and spacelike normals to a sphere of radius $r$.

Since $f(r)$ does not contribute to the equations for $g(r), h(r)$ and $j(r)$ we can first study the set of equations involving $\{g, h, j\}$. Inserting the perturbative expansion (4.1) into equations (2.12-2.14), we obtain the equations for small perturbations

$$
\begin{gathered}
r \frac{d}{d r}\left(\begin{array}{c}
\delta g \\
\delta h \\
\delta j
\end{array}\right)=\left(\begin{array}{ccc}
-n & z(z-1) /(n-1) & z-1 \\
n-1 & 1-n & n-1 \\
-(n+z-2) & z(n+z-2) /(n-1) & 1-2 z
\end{array}\right)\left(\begin{array}{l}
g_{1} \\
h_{1} \\
j_{1}
\end{array}\right) \\
+\left(\frac{Q^{2}}{4(n-1) r^{2 n-2}}+\frac{(n-2) k}{2 r^{2}}\right)\left(\begin{array}{l}
1 \\
0 \\
1
\end{array}\right)
\end{gathered}
$$

where we have included the Maxwell gauge field as a first order perturbation which means that we substitute $Q^{2} / r^{2(n-1)}=w Q^{2} / r^{2(n-1)}$, since its falloff may be slower than other terms in the metric functions. We have also rescaled $r \rightarrow r / \ell$. Note that for $k \neq 0$ a universal $1 / r^{2}$ mode also contributes to the metric functions. 
A detailed discussion of the large $r$ expansion is given in the appendix. The eigenvalues of the matrix of coefficients is obtained via straightforward calculation

$$
\left(\begin{array}{c}
z+n-1 \\
\frac{z+n-1}{2}+\frac{\sqrt{9 z^{2}-2(3 n+1) z+\left(n^{2}+6 n-7\right)}}{2} \\
\frac{z+n-1}{2}-\frac{\sqrt{9 z^{2}-2(3 n+1) z+\left(n^{2}+6 n-7\right)}}{2}
\end{array}\right)
$$

indicating there are three independent eigenmodes.

The two upper modes in (4.4) are always decaying. However the lowest mode, depending on the value of $z$, will either be a growing mode if $z>n-1$, a zero mode (independent of $r$ ) if $z=n-1$, or a decaying mode if $z<n-1$. Then if one looks for the solutions that asymptotically approach a Lifshitz fixed point, one has to discard the lowest mode in (4.4) for $z \geq n-1$ by demanding the amplitude of this mode vanishes at large $r$.

Removing zero or growing modes can be done numerically in both uncharged or charged solutions by fine-tuning initial values upon solving the field equations. It is obvious that one has to remove the growing mode for $z>n-1$. The zero mode for $z=n-1$ becomes a marginally growing mode when non-linear corrections are included [8]. Such a mode with positive amplitude cannot approach the Lifshitz geometry (1.2) asymptotically and therefore must be removed. On the other hand, a zero mode with negative amplitude will slowly decay; in this case a consideration of the evolution of $f(r)$, (ignored thus far), indicates that it goes to zero as $r \rightarrow \infty$ again yielding unacceptable asymptotic behaviour.

For $z<n-1$ all three eigenmodes decay, and so no fine-tuning of initial values is required if one simply wishes to obtain solutions that asymptote to the Lifshitz metric (1.2). For a given event horizon size we have a family of solutions that are all asymptotic to the Lifshitz metric (1.2), but with different fall-off rates. By an appropriate choice of boundary terms, such solutions will have finite energy [20, 21].

\section{Near horizon expansion}

In order to investigate the near horizon behavior of the solutions, consider the following expansions for the fields:

$$
\begin{aligned}
& f(r)=f_{0} \sqrt{r-r_{0}}\left(1+f_{1}\left(r-r_{0}\right)+f_{2}\left(r-r_{0}\right)^{2}+\cdots\right), \\
& g(r)=\frac{g_{0}}{\sqrt{r-r_{0}}}\left(1+g_{1}\left(r-r_{0}\right)+g_{2}\left(r-r_{0}\right)^{2}+\cdots\right), \\
& j(r)=j_{0} \sqrt{r-r_{0}}\left(1+j_{1}\left(r-r_{0}\right)+j_{2}\left(r-r_{0}\right)^{2}+\cdots\right), \\
& h(r)=h_{0}\left(1+h_{1}\left(r-r_{0}\right)+h_{2}\left(r-r_{0}\right)^{2}+\cdots\right),
\end{aligned}
$$



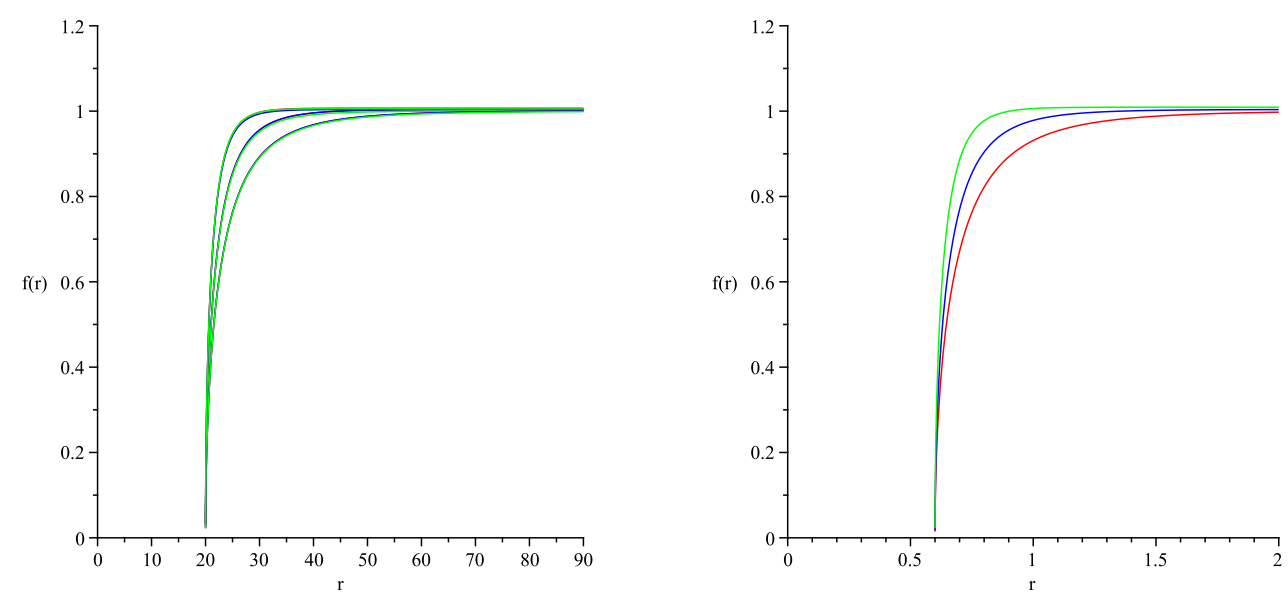

Figure 2: Metric functions $f(r)$ for zero modes in 4, 5 and 7-dimensions from bottom to top respectively. Left) $r_{0}=20$ for all $k$. Right) $r_{0}=0.6$ for $k=0$.

where $r_{0}$ is the horizon radius. All other constants in the series solutions (5.1) can be obtained in terms of $r_{0}$ and $h_{0}$ as well but we omit them here because they are too lengthy.

Inserting this into eqs. (2.11-2.14) and demanding the coefficients for each power of $r-r_{0}$ vanish determines all constants in terms of $r_{0}$ and $h_{0}$. For example $g_{0}$ is given by

$$
g_{0}=r_{0}^{1 / 2} \sqrt{\frac{2(n-1)}{r_{0}^{2-2 n}\left(Q_{c}^{2}-Q^{2}\right)-2 z(z-1) h_{0}^{2}}}
$$

where

$$
Q_{c}^{2} \equiv \frac{2}{r_{0}^{4-2 n}}\left\{\left[(z-1)^{2}+(z-2) n+n^{2}\right] r_{0}^{2}+(n-1)(n-2) k \ell^{2}\right\}
$$

Requiring $g_{0}$ to be real we obtain the constraint

$$
|Q| \leq \sqrt{Q_{c}^{2}-2 z(z-1) h_{0}^{2} r_{0}^{2 n}}
$$

generalizing a similar constraint obtained for $Q=0$ black holes [8, 9]. Indeed, for $Q=0$ the constraint (5.4) reduces to

$$
\left[(z-1)^{2}+(z-2) n+n^{2}-z(z-1) h_{0}^{2}\right] r_{0}^{2}+(n-1)(n-2) k \ell^{2} \geq 0
$$

imposing either an upper bound on $\left|h_{0}\right|$ if $k=0,1$ or imposing a lower bound of $r_{0}>\sqrt{\frac{(n-1)(n-2)}{(z-1)^{2}+(z-2) n+n^{2}}}$ if $k=-1$.

For $Q \neq 0$ equation (5.4) is more usefully written as

$$
\frac{(z-1)^{2}+(z-2) n+n^{2}-z(z-1) h_{0}^{2}}{(n-1)(n-2)} r_{0}^{2}+k \ell^{2} \geq \frac{Q^{2}}{2 \ell^{2}(n-1)(n-2) r_{0}^{2(n-1)}}
$$



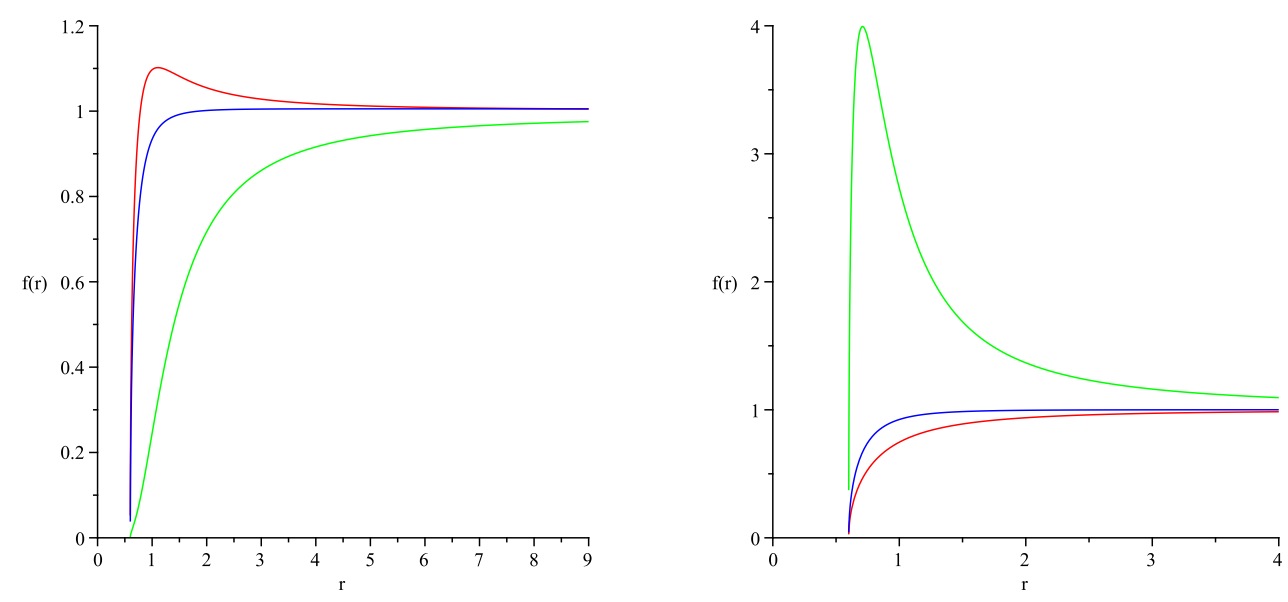

Figure 3: Metric functions $f(r)$ for zero modes with $r_{0}=0.6$ in 4 (red), 5 (blue) and 7 (green)-dimensions for: Left) $k=1$; Right) $k=-1$.

or alternatively as $\alpha X+k>X^{1-n}$, where

$$
\alpha=\frac{(z-1)^{2}+(z-2) n+n^{2}-z(z-1) h_{0}^{2}}{(n-1)(n-2)}\left(\frac{Q^{2} \ell^{-2(n+1)}}{2(n-1)(n-2)}\right)^{\frac{1}{n-1}}
$$

upon setting $r_{0}^{2}=X \ell^{2}\left(\frac{Q^{2} \ell^{-2(n+1)}}{2(n-1)(n-2)}\right)^{\frac{1}{n-1}}$. We see that analysis of this equation involves finding the intersection points of a straight line in $X$ with a curve behaving as $X^{1-n}$. The line intersects the origin for $k=0$, and has an intercept at \pm 1 for $k= \pm 1$. For $h_{0} \rightarrow 0$ the line always intersects the $X^{1-n}$ curve, and so any values of $X$ larger than this are admissible. Hence for any given $Q$ there is a lower bound on the size of the black hole regardless of the value of $k$. The lower bound is largest for $k=-1$ and smallest for $k=1$. As $h_{0}$ increases the slope of the line decreases, and so this lower bound increases. For $k=0,-1$ it becomes infinite as $\alpha \rightarrow 0$, i.e. $h_{0} \rightarrow \frac{(z-1)^{2}+(z-2) n+n^{2}}{z(z-1)}$. Indeed for small $\alpha$ we find

$$
X \geq \frac{1}{\alpha}\left(1-(n-1) \alpha^{n-1}-\frac{(n-2)(n-1)}{2} \alpha^{2 n-2}+\frac{\left(4 n^{2}-8 n+3\right)(n-1)}{3} \alpha^{3 n-3}+\cdots\right)
$$

and we see that the lower bound on $X$ diverges as $\alpha \rightarrow 0$.

However for $k=1$ the situation is quite different. As $h_{0} \rightarrow \frac{(z-1)^{2}+(z-2) n+n^{2}}{z(z-1)}$ the lower bound on $X$ remains finite. As $h_{0}$ becomes even larger, a larger lower bound on $X$ appears since now $\alpha<0$. As $h_{0}$ increases, $\alpha$ becomes more negative, and the lower bound on $X$ continues to decrease. Eventually a limit of $X>n^{\frac{1}{n-1}}$ is reached at which the line is tangent to the $X^{1-n}$ curve, where $\alpha=(1-n) n^{\frac{n}{1-n}}$, or

$$
\frac{z(z-1) h_{0}^{2}-(z-1)^{2}+(z-2) n+n^{2}}{(n-1)^{2}(n-2)}=\left(\frac{Q^{2} n^{n} \ell^{-2(n+1)}}{2(n-1)(n-2)}\right)^{\frac{1}{1-n}}
$$


For values of $h_{0}$ larger than this, no black hole solutions exist; instead there is a naked singularity.

Alternatively if we fix $r_{0}$ then demanding $g_{0}$ to be real implies $Q_{c}^{2}>0$ (and so $\left.|Q|<\left|Q_{c}\right|\right)$ as well as

$$
\left|h_{0}\right|<\sqrt{\frac{\ell^{2} r_{0}^{2-2 n}\left(Q_{c}^{2}-Q^{2}\right)}{2 z(z-1) L^{4}} .} .
$$

Positivity of $Q_{c}^{2}$ is always satisfied if $k=0,1$. However for $k=-1$ it is satisfied provided $r_{0}$ respects a lower bound as

$$
r_{0}>\sqrt{\frac{(n-1)(n-2)}{n^{2}+n(z-2)+(z-1)^{2}}} .
$$

The value $Q \rightarrow Q_{c}$ is the extremal limit of the charged Lifshitz black hole [12, 13], defined in eq. (5.3); it corresponds to the value of $Q$ for which the temperature vanishes.

\section{Numeric Solutions in $(n+1)$-dimensions}

In order to find numeric solutions for the system of ODE's (2.11-2.14) we apply the shooting method by adjusting some initial values for the fields $f(r), g(r), j(r)$ and $h(r)$. To find the initial values we employ the near horizon expansions (5.1) by substituting $r_{0}+\varepsilon$ for $r$ where $\varepsilon \ll 1$, choosing some values for $r_{0}$ and $h_{0}$ such that after applying numerical method the metric and field functions approach unity at large $r$.

\subsection{Uncharged Einstein-Lifshitz Solutions}

In order to study uncharged Einstein-Lifshitz solutions we put $Q=0$ in ODE's (2.112.14) and use the numerical method to find the solutions. We already explained there are different situations depending on the value of critical exponent $z$ :

I) We begin with an examination of situations where the zero mode is present, which implies $z=n-1$. For a given horizon radius $r_{0}$ the solution is unique, requiring a fine-tuning of initial values to approach the Lifshitz metric (1.2) at large $r$. We find that the solutions are similar to the $n=3$ case [9]: for each $n$, when $k=0$ the same value of $h_{0}$ yields black hole solutions for all values of $r_{0}$. Specifically, $h_{0}=1.3737,1.3344,1.3084$ in 4,5 and 7 dimensions respectively. For $k=1(-1)$ it is necessary to systematically adjust the value of $h_{0}$ upward (downward) relative to the value for $k=0$. Also, for each $n$ large black holes are almost indistinguishable for different choices of $k$, see Fig. (2-left). Distinctions start to appear between solutions of the same dimension but different $k$ as the horizon radius $r_{0}$ gets smaller.

We can also probe the behaviour of our solutions as a function of dimensionality. Looking at the left-hand side of Fig. (2), we see that for large $r_{0}$, where the solutions 

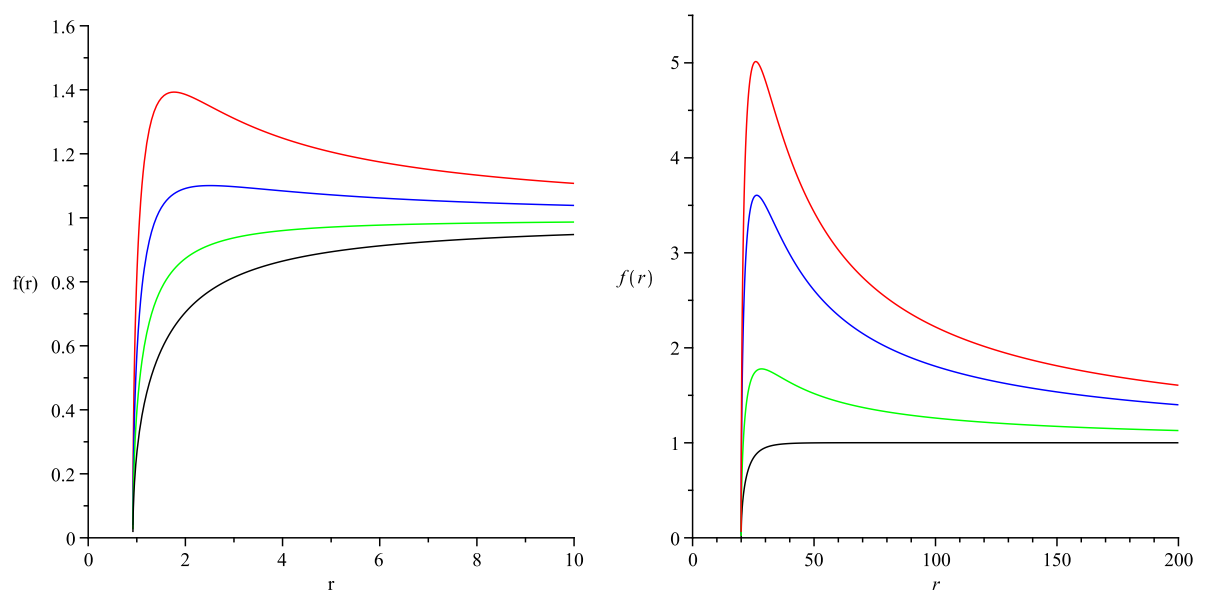

Figure 4: Metric functions $f(r)$ for $k=-1$ in 7-dimensions with $z=2$ : Left) $r_{0}=$ 0.92. Right) $r_{0}=20$ while the massive gauge field strength $h_{0}$ increases and temperature decreases from top to bottom in both cases.

are almost the same different values of $k$, as the dimension increases the solutions approach (1.2) more rapidly as $r$ increases. However the overall behaviour of the solutions is essentially the same: the metric function $f(r)$ starts from zero at the horizon radius and monotonically increases to asymptotically approach unity at large $r$. The situation is the same for small black holes when $k=0$, shown at the right in Fig. (2).

However, for small black holes with $k=1$ or $k=-1$ the situation differs, as depicted in Fig. (3). For $k=1$ in 4-dimensions, the metric function $f(r)$ starts from zero at the horizon radius increasing to reach a maximum above unity, then decreasing to approach unity at large $r$. However in dimensions greater than 4, the metric function $f(r)$ starts from zero at the horizon radius and monotonically increases to asymptotically approach unity as $r$ goes to infinity. For $k=-1$ the situation is completely vice-versa. Examples are shown in Fig. (3), with $k=1$ on the left and $k=-1$ on the right.

II) $z<n-1$ : For the series solutions at large $r$ all three eigenmodes resultant from the small perturbation are decaying, and so no fine-tuning is required. In this case it is possible to find solutions that all approach unity at large $r$ by fixing $r_{0}$ and then searching for a value of $h_{0}$ such that the boundary conditions are satisfied. We find that $h_{0}$ is not unique; in other words there is a continuous spectrum of values of $h_{0}$ that all respect the boundary conditions. Numerical exploration shows that for strong massive gauge field, i.e. a larger value of $h_{0}$, the metric function $f(r)$ starts from zero at the horizon radius and monotonically increases to asymptotically reach unity. However, for a smaller value of $h_{0}, f(r)$ starts from zero at the horizon radius and as $r$ increases, passes through a maximum value greater than unity, and then approaches unity as $r$ goes to infinity. Moreover, black holes with stronger massive 

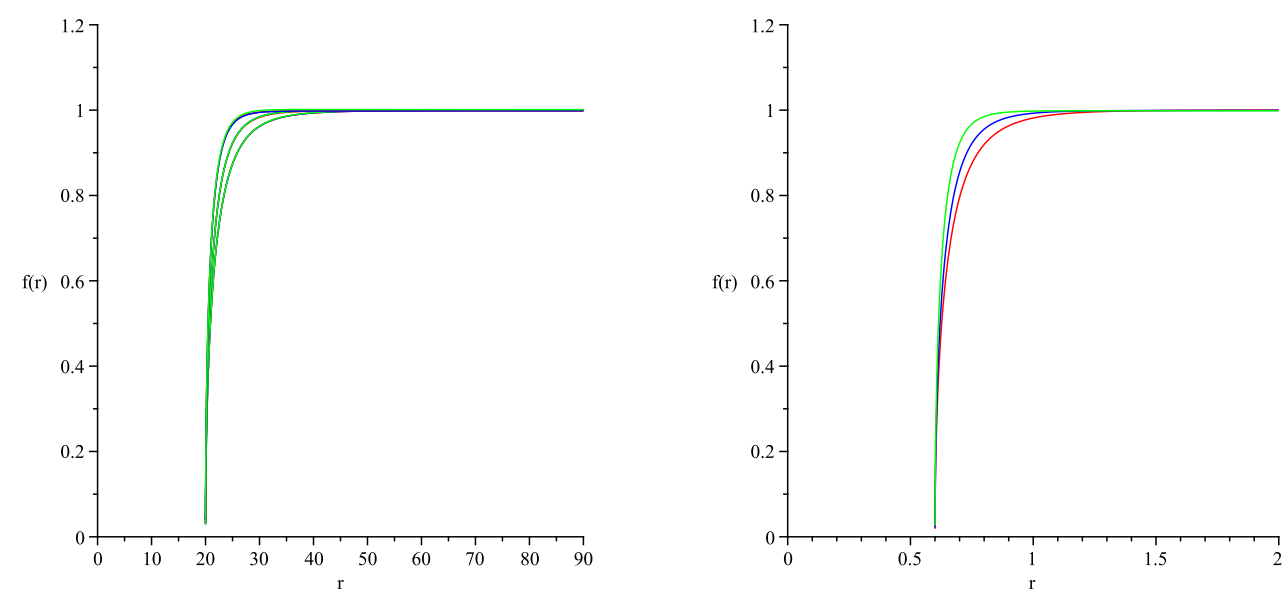

Figure 5: Metric functions $f(r)$ in 4, 5 and 7-dimensions with $z=4,5$ and 7 from bottom to top, respectively. Left) $r_{0}=20$ and all values of $k$ 's. For a given dimension the different values of $k$ lie almost exactly on the same curve. Right) $r_{0}=0.6$ and $k=0$.
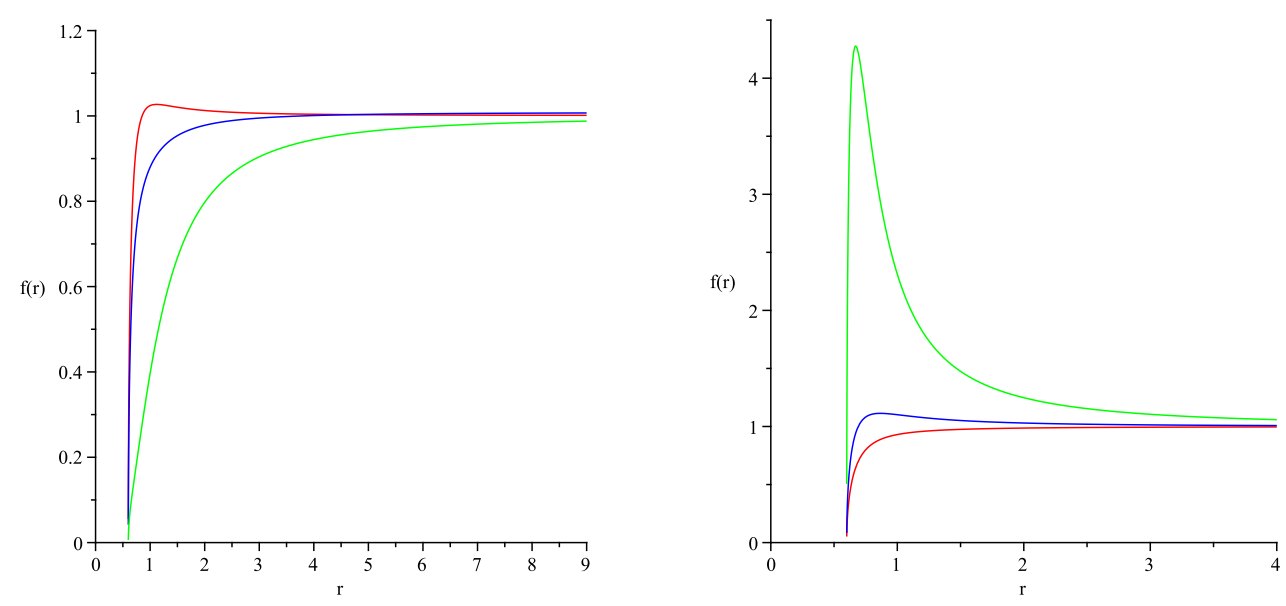

Figure 6: Metric functions $f(r)$ with $r_{0}=0.6$ in 4 (red), 5 (blue) and 7 (green)-dimensions and $z=4,5$ and 7 respectively. Left) $k=1$. Right) $k=-1$.

gauge field are colder than those with weaker ones. For example, in 7 dimensions with $z=2$ and $k=-1$ then, for a fixed $r_{0}$, there is a family of solutions that have qualitatively different behaviour compared to each other but all are asymptotic to the Lifshitz background at large $r$. An illustration of this is in Fig. (4) for both small and large black holes. We find the same situation in other dimensions with different choices of $z$ and $k$ provided $z<n-1$.

III) $z>n-1$ : In this case one eigenmode amongst three is growing. To have solutions asymptotic to (1.2) the initial values of the parameters must be adjusted such that the amplitude of this mode is removed otherwise the fields diverge so fast at large $r$. Compared to the zero mode the fine-tuning is numerically more delicate - approaching (1.2) at large $r$ needs to more accuracy in digits of $h_{0}$. However, the 

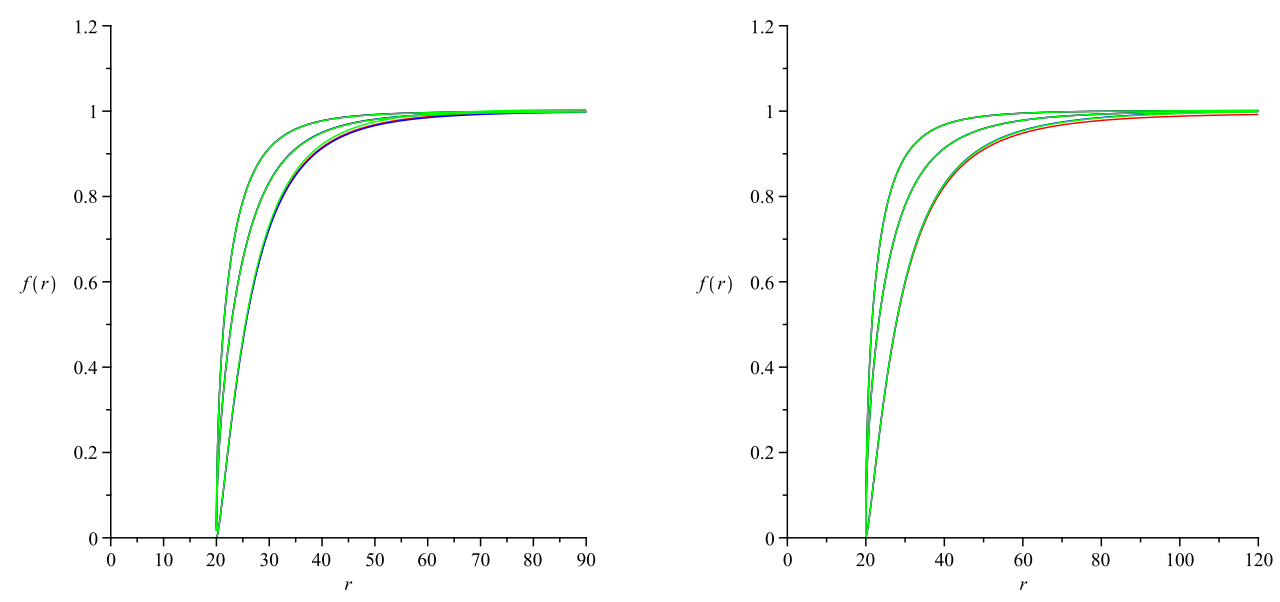

Figure 7: Metric functions $f(r)$ for $r_{0}=20, z=2$ and all $k$. Left) in 5 dimensions and $Q=0,30000,46600$ from top to bottom $\left[Q_{c} \approx 46600\right]$. Right) in 4 dimensions and $Q=0,1100,1780$ from top to bottom $\left[Q_{c} \approx 1788\right]$. In each case $k=0$ is red, $k=1$ is blue and $k=-1$ is green; these different cases are just barely distinguishable for such large values of $r$.

results are similar to the zero mode case: for each $n$ large black holes are almost indistinguishable for different choices of $k$ and the metric function $f(r)$ reaches unity faster as the dimension of spacetime increases, as shown in the left side of Fig. (5). Distinctions start to appear between solutions of the same dimension but different $k$ as the horizon radius $r_{0}$ gets smaller, illustrate in the right side of Figs. (5) and in Fig. (6).

\subsection{Charged Einstein-Lifshitz Solutions}

We now consider how the Maxwell gauge field changes the solutions. First, the requirement [13] that the field strength $\left(r^{z} \kappa\right)^{\prime}$ of the massless gauge field vanishes as $r$ goes to infinity implies $z \leq(n-1)$. For the zero mode case $(z=n-1)$, we find that if $Q \ll Q_{c}$ these solutions are not significantly different from uncharged ones. However distinctions start to appear as the Maxwell charge $Q$ becomes comparable to $Q_{c}$. Indeed in each dimension, for large black holes both $Q_{c}$ and the metric/gauge functions are almost indistinguishable for differing values of $k$ and the same charge, as shown in Fig. (7). This is not the case for small black holes. Here $Q_{c}$ significantly depends on $k$ and the solutions are distinguishable for differing $k$. However in general, as $Q$ increases the solutions either approach unity at large $r$ less rapidly or become less sharply peaked for intermediate values of $r$ in situations where such peaks exist. For example, three different solutions for small black holes of the same radius but different Maxwell charges are illustrated in Fig. (8). The left-hand side of the diagram is for $k=1$ in 4 -dimensions and the right-hand side is for $k=-1$ in 7-dimensions. 

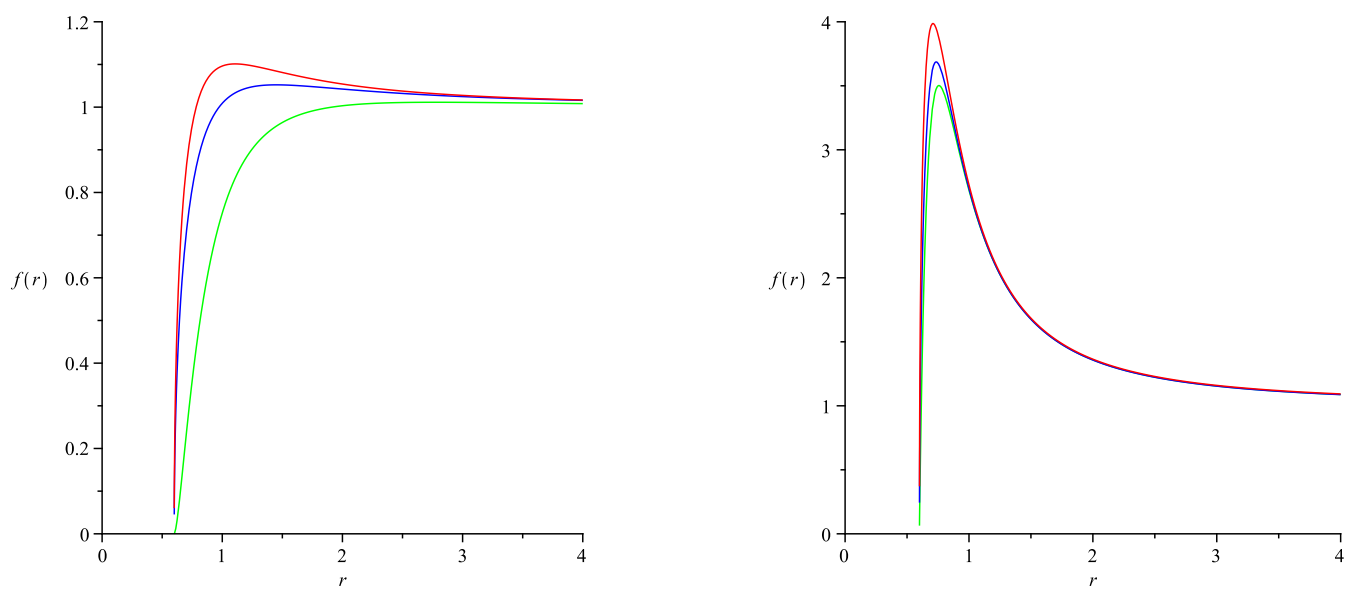

Figure 8: Metric functions $f(r)$ for different dimensions. Left) 4-dimensions with $r_{0}=0.6$ for zero mode and $k=1$ for $Q=0,1,2$ from top to bottom, respectively [ $\left.Q_{c} \approx 2\right]$. Right) 7-dimensions with $r_{0}=0.6$ for zero mode and $k=-1$ for $Q=0,0.3,0.41$ from up to down, respectively $\left[Q_{c} \approx 0.417\right]$. Increasing values of $Q$ are coloured red, blue, and green respectively in each case.
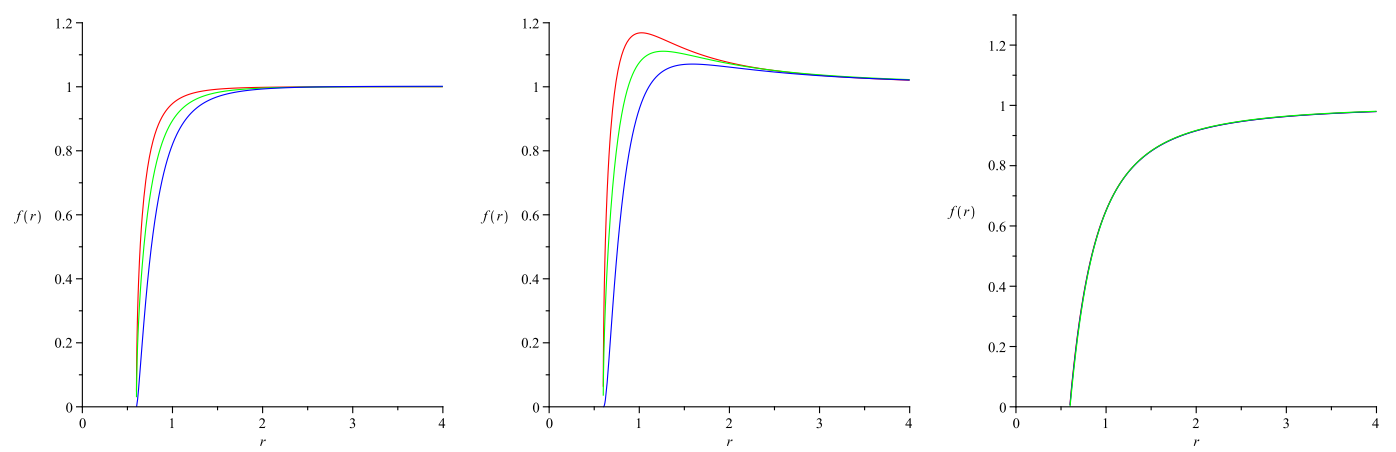

Figure 9: metric functions $f(r)$ in 5 dimensions for $r_{0}=0.6$ and $z=2$ for: Left) $k=0$ and $Q=0,0.8,1.259\left[Q_{c} \approx 1.259\right]$, Middle) $k=1$ and $Q=0,1.1,1.77\left[Q_{c} \approx 1.77\right]$, Right) $k=-1$ and $Q=0,0.1,0.176\left[Q_{c} \approx 0.176\right]$ from top to bottom, respectively. Increasing values of $Q$ are coloured red, blue, and green respectively in each case.

If $z<n-1$, for a fixed $r_{0}$ there is a family of solutions depending on fall-off rate. Consequently to investigate how the Maxwell charge modifies the solutions by demanding the same fall-off rate (for a given value of $k$ ) we search for possible charged black holes. Numerical exploration reveals that with a fixed $r_{0}$, we cannot find a solution if $Q>Q_{c}$, where $Q_{c}$ is given by eq. (5.3). Fig. (9) shows $f(r)$ for small black holes in 5 dimensions with $z=2$ and $k=0,1,-1$ for different $Q$.

Corresponding plots can be constructed for $g(r)$ and $h(r)$; we have not reproduced them here. 

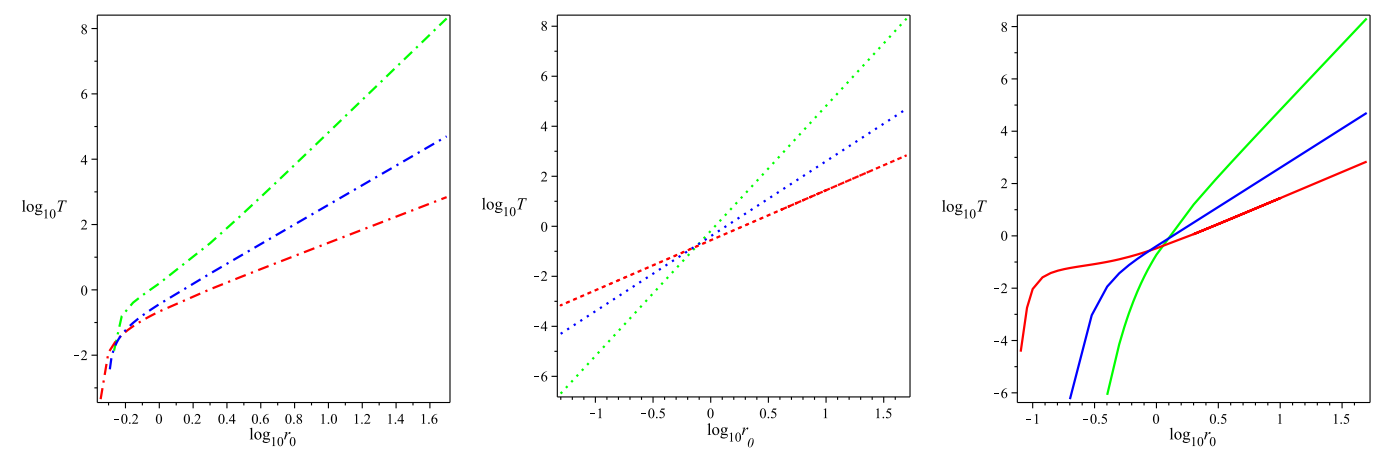

Figure 10: Temperature vs $r_{0}$ with $Q=0$ for zero modes in 4 (red), 5 (blue) and 7 (green)dimensions for: $k=-1$ (dash-dots), $k=0$ (dots) and $k=1$ (solids).

\section{Thermal behavior}

The temperature of Lifshitz black holes is easily evaluated using standard Wick rotation methods, yielding the result

$$
T=\frac{f_{0} r_{0}^{z+1}}{4 \pi g_{0}}
$$

where $g_{0}$ is given by (5.2) and $f_{0}$ is determine so that the metric has asymptotic behavior given in equation (1.2). The temperature clearly depends on the spacetime dimensionality $(n+1)$, the critical exponent $z$, the topological parameter $k$, the horizon radius $r_{0}$, and the Maxwell charge $Q$.

The behavior of temperature versus $r_{0}$ for uncharged solutions $Q=0$ and zero modes $z=n-1$ in different dimensions is depicted in Fig. (10) for $k=-1,0$ and 1. For all topologies we see that the temperature increases with dimensionality for large black holes, whereas it decreases with dimensionality for small black holes. This effect is most pronounced for $k=1$, and least so for $k=-1$. In each dimension the temperature decreases as the horizon radius shrinks, i.e. small black holes are colder than large ones. For $k=-1$, there is a lower bound on the radius of the black hole.

For $Q \neq 0$, since the metric functions $f(r)$ and $g(r)$ are not altered significantly when $Q$ is small compared to its upper bound $Q_{c}$ no significant change in the behaviour of the temperature is expected. This upper bound value increases with increasing horizon radius. Indeed, with a fixed horizon the temperature goes to zero as $Q \rightarrow Q_{c}$. We therefore consider the behaviour of temperature as a function of $Q$ for fixed horizon radius $r_{0}$.

Figure (11) illustrates the situation in 4-dimensions for both small $\left(r_{0}=0.6\right)$ and large $\left(r_{0}=20\right)$ black holes. For all topologies the temperature decreases with increasing $Q$ as expected, with temperature being largest for the spherical case and smallest for the hyperbolic one. For large black holes this latter effect is almost completely indistinguishable, whereas for small black holes it is quite pronounced. 

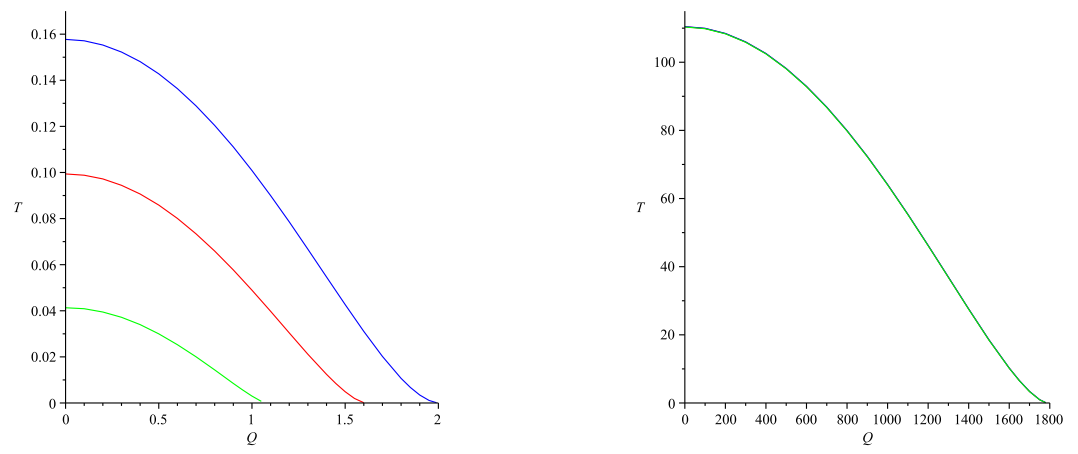

Figure 11: Temperature vs $Q$ in 4-dimensions with $z=2$ for: $r_{0}=0.6$ for $k=-1$ (green), $k=0$ (red) and $k=1$ (blue) on the left and $r_{0}=20$ on the right; in the latter case the curves are almost identical for each $k$.
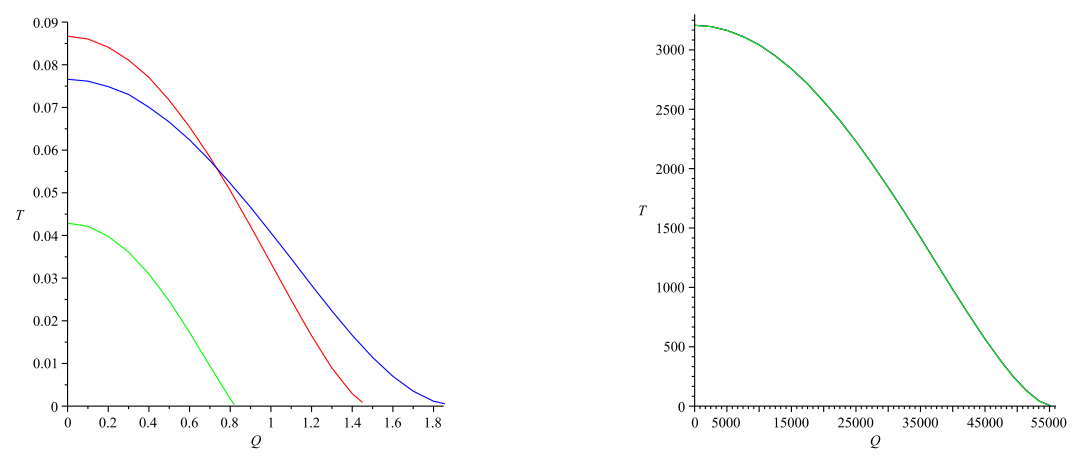

Figure 12: Temperature vs $Q$ in 5-dimensions with $z=3$ for: $r_{0}=0.6$ for $k=-1$ (dash-dot), $k=0$ (dot) and $k=1$ (solid) on the left and $r_{0}=20$ for all $k$ on the right; in the latter case the curves are almost identical for each $k$.

Furthermore, this effect does not hold as dimensionality increases. In Fig. (12) we plot temperature versus $Q$ in 5-dimensions for both small and large black holes. In the former case we see that for sufficiently large $Q$ flat topologies yield hotter black holes than spherical ones. In the latter case the distinctions between topologies are very small - they exhibit nearly the same temperature for different $k$, a feature we find true in all dimensions we have checked. In any dimension the temperature approaches zero as the Maxwell charge approaches its extremal value.

\section{Wilson loop}

In 4 dimensions the action for the dual theory is conjectured to be

$$
S=\int d t d^{2} \mathbf{x}\left(\dot{\phi}^{2}-K\left(\nabla^{2} \phi\right)^{z}\right)
$$

and if we write $\nabla^{2} \phi=\vec{\nabla} \times \vec{E}$, where $E_{j}=\varepsilon_{j k} \nabla^{k} \phi$, then the boundary action (8.1) could be regarded as a gauge theory in $(2+1)$ dimensions with a dimensionless 


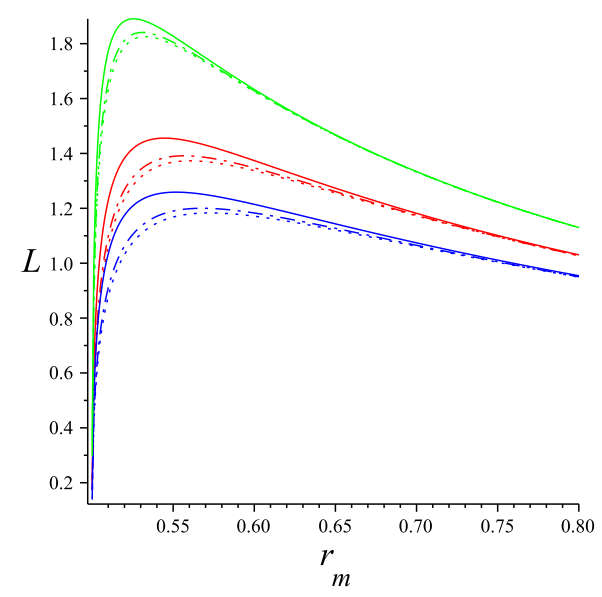

Figure 13: Boundary length $L$ vs $r_{m}$ in 4-dimensions with $z=2$ and $r_{0}=0.5$ for $k=1$ (blue), $k=0$ (red) and $k=-1$ (green) while in each case dot is for $Q=0$, dash-dot for $Q=Q_{c} / 3$ and solid for $Q=2 Q_{c} / 3$.

coupling constant $[8,7]$. We introduce Wilson loops by joining charged particles on the boundary that are connected together in the bulk via a string. These loops contain information about the force acting between particles charged under the gauge fields in the dual theory.

The Euclidean action of this string for a rectangular Wilson loop is the same for all values of $k$ and is given by [7, 19]

$$
\mathcal{S}=\frac{1}{2 \pi \alpha^{\prime}} \int d t d \tau \sqrt{\operatorname{det}\left[g_{A B} \partial_{\mu} X^{A} \partial_{\nu} X^{B}\right]}=\frac{\triangle \ell^{2}}{2 \pi \alpha^{\prime}} \int d \theta \sqrt{f^{2} r^{2 z+2}+f^{2} g^{2} r^{2 z-2}\left(\frac{d r}{d \theta}\right)^{2}}
$$

taking $\sigma=\theta$ and $i \tau=t$ in the static gauge, with Euclidean time interval $\triangle$.

If one extremizes the action one may obtains a constant of the motion as

$$
\frac{f^{2} r^{2 z+2}}{\sqrt{f^{2} r^{2 z+2}+f^{2} g^{2} r^{2 z-2}\left(\frac{d r}{d \theta}\right)^{2}}}=f\left(r_{m}\right) r_{m}^{z+1}
$$

where $r_{m}>r_{0}$ is the location of the midpoint of the string for which $\left.\frac{d r}{d \theta}\right|_{r_{m}}=0$. From the above expression one may get the boundary length as

$$
L=\int d \theta=2 \int_{r_{m}}^{\infty} \frac{d r}{r^{2}} \frac{g}{\sqrt{\left(\frac{f}{f_{m}}\right)^{2}\left(\frac{r}{r_{m}}\right)^{2 z+2}-1}}
$$

and the regularized potential energy between the two particles

$$
V=\frac{\mathcal{S}}{\triangle \ell}=\frac{\ell}{2 \pi \alpha^{\prime}}\left(2 \int_{r_{m}}^{\infty} d r \frac{r^{z-1} f g}{\sqrt{1-\left(\frac{f}{f_{m}}\right)^{2}\left(\frac{r_{m}}{r}\right)^{2 z+2}}}-2 \int_{r_{0}}^{\infty} d r r^{z-1} f g\right)
$$



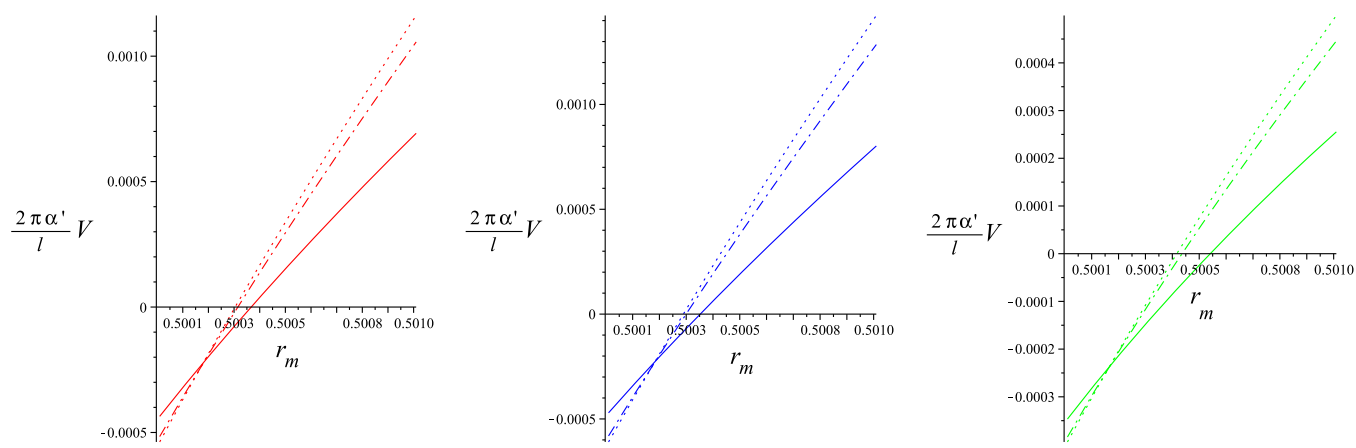

Figure 14: Potential $V$ between two particles as a function of string midpoint $r_{m}$ in 4dimensions with $z=2$ and $r_{0}=0.5$ for $k=0$ (red), $k=1$ (blue) and $k=-1$ (green) for $Q=0$ (dot), $Q=Q_{c} / 3$ (dash-dot) and $Q=2 Q_{c} / 3$ (solid).

where $f_{m}=f\left(r_{m}\right)$.

We plot in Fig. (13) how the behaviour of the Wilson loop for the neutral case $[8,9]$ is modified for nonzero $Q$. For a given $r_{m}$ we find that the boundary length increases and the potential between objects in the dual theory decreases (Fig. (14)) with increasing Maxwell charge.

\section{Conserved Charge}

To explore the conserved charge we redefine the metric and gauge fields as follows while we have chosen $\ell=1$ for simplicity through the rest of the paper:

$$
\begin{aligned}
& d s^{2}=-e^{2 F(r)} d t^{2}+e^{2 G(r)} d r^{2}+e^{2 R(r)} d \mathbf{x}^{2}, \\
& A_{t}=e^{K(r)}, \quad B_{t}=q e^{J(r)}, \quad H_{t r}=q e^{H(r)} .
\end{aligned}
$$

where new fields $F, G, R, K, J, H$ can be expressed in terms of the old fields as:

$$
\begin{aligned}
& F(r)=\ln \left(r^{z} f\right), \quad G(r)=\ln (g / r), \quad R(r)=\ln r, \\
& H(r)=\ln \left(z r^{z-1} g h f\right), \quad J(r)=\ln \left(r^{z} j f\right), \quad K(r)=\ln \left(r^{z} \kappa\right) .
\end{aligned}
$$

If one inserts the ansatz (9.1) into the action (1.3), one obtains the one dimensional lagrangian $\mathcal{L}_{1 D}=\mathcal{L}_{1 g}+\mathcal{L}_{1 m}$ with

$$
\begin{aligned}
\mathcal{L}_{1 g} & =(n-1)\left[-\frac{2 \Lambda e^{2 G}}{n-1}+2 F^{\prime} R^{\prime}+(n-2) R^{\prime \prime}\right] e^{F-G+(n-1) R}, \\
\mathcal{L}_{1 m} & =\frac{1}{2} e^{-F+G+(n-1) R+2 J}\left[q^{2}\left(C+J^{\prime 2} e^{-2 G}\right)+K^{\prime 2} e^{2 K-2 J-2 G}\right] .
\end{aligned}
$$

where we have used eq. (2.3) to write $H(r)$ in terms of $J(r)$. Applying the same method described in [17] reveals that

$$
\begin{aligned}
\mathcal{C} & \equiv 2\left(F^{\prime}-R^{\prime}\right) e^{F-G+(n-1) R}-\left(q^{2} J^{\prime} e^{2 J}-K^{\prime} e^{2 K}\right) e^{-F-G+(n-1) R} \\
& =\frac{r^{n+z-1}}{g}\left[2(z-1) f+2 r f^{\prime}-q^{2} j\left(z f j+r f j^{\prime}+r j f^{\prime}\right)\right]-Q r^{z} \kappa,
\end{aligned}
$$


is conserved along the radial coordinate $r$ and

$$
\kappa(r)=\frac{1}{r^{z}}\left[Q \int^{r} \frac{f g}{r^{n-z}} d r+D\right]
$$

where $D$ is an integration constant, which is chosen such that $\kappa(r)$ vanishes at the horizon, that is

$$
D=-Q \int^{r_{0}} \frac{f g}{r^{n-z}} d r
$$

We shall evaluate $\mathcal{C}$ both at the horizon radius and infinity. Now using (9.6) and the large $r$ expansions (11.1), (11.2), (11.3) and (11.8) conserved charge for $z \neq n-1$ is given by:

$$
\mathcal{C}=\frac{2(z-1)(n-z-1)(z+n-1)}{(n-1)^{2}} C_{1}-Q D .
$$

and for $z=n-1$ is:

$$
\mathcal{C}=\frac{Q^{2}}{2(n-1)}-\frac{2 C_{1}}{n-1}
$$

Using the near horizon expansions (5.1) we find that

$$
\mathcal{C}=\frac{f_{0}}{g_{0}} r_{0}^{n+z}=16 \pi T S
$$

while we have considered massless field is zero at the horizon and $S$ is the entropy of the black brane per unit volume which in terms of the horizon radius is given by:

$$
S=\frac{1}{4} r_{0}^{n-1} .
$$

We remark that the conserved quantity $\mathcal{C}$ for $z=1$ with $g(r)=1 / f(r)$ reduces to

$$
\mathcal{C}=2 r^{n+1} f f^{\prime}+\frac{Q^{2}}{(n-2) r^{n-2}},
$$

which is proportional to the mass of the asymptotically AdS-Reissner-Nordstrom solution and gives the metric function as

$$
f^{2}(r)=1-\frac{m}{r^{n}}+\frac{Q^{2}}{2(n-1)(n-2) r^{2(n-1)}},
$$

which is the well-known asymptotically AdS Reissner-Nordstrom solution with flat horizon.

\section{Finite Action and the Energy Density for Einstein-Maxwell- Lifshitz Solutions}

In order to have a finite action we must add some boundary terms to the original bulk action. For this purpose, we use the same method proposed in [20] to make 
$(n+1)$-dimensional Einstein-Maxwell-Lifshitz(EML) action finite and well-defined. We consider $I=I_{\text {bulk }}+I_{\text {bdy }}$, where $I_{\text {bulk }}$ is given in eq. (1.3) and $I_{\text {bdy }}$ is the boundary action which for the case of zero curvature boundary can be written as:

$I_{\mathrm{bdy}}=\frac{1}{8 \pi} \int_{\partial \mathcal{M}} d^{n} x \sqrt{-h}\left[K-(n-1)+\frac{1}{2} f\left(B_{\alpha} B^{\alpha}\right)\right]+\frac{\omega}{8 \pi} \int_{\partial \mathcal{M}} d^{n} x \sqrt{-h} n^{\mu} F_{\mu \nu} A^{\nu}+I_{\text {deriv }}$

where in the first integral, which is added to cancel the divergences from gravity and the Lifshitz field, the boundary $\partial \mathcal{M}$ is the hypersurface at some constant $r, h_{\alpha \beta}$ is the induced metric, $K$ is the trace of the extrinsic curvature, $K_{\alpha \beta}=\nabla_{(\alpha} n_{\beta}$ of the boundary (where the unit vector $n^{\mu}$ is orthogonal to the boundary and outwarddirected).

In the grand canonical ensemble we set $\delta A^{\mu}=0$ on the boundary and the variational principle is well-defined only if $\omega=0$. In the canonical ensemble we hold the electric charge (given by eq. (4.2)) fixed, which is equivalent to setting $\delta\left(\sqrt{-h} n^{\mu} F_{\mu \nu}\right)=0$ on the boundary [24]. In this latter instance the variational principle is well-defined provided we select $\omega=\frac{1}{2}$. The last term $I_{\text {deriv }}$ in (10.1) is a collection of terms involving derivatives of the boundary fields, which could involve both the curvature tensor constructed from the boundary metric and covariant derivatives of $B_{\alpha}$. Since the boundary is flat and the fields are constants for (1.2), this term will not contribute to the on-shell value of the action for the pure Lifshitz solution or its first variation around the Lifshitz background and therefore we ignore it henceforth. As explained in [20], an arbitrary function $f\left(B^{\alpha} B_{\alpha}\right)$ is added to the action which is due to the fact that on the boundary $B_{\alpha} B^{\alpha}=-q^{2}$ is constant for Lifshitz solutions.

The variation of the action about a solution of the equations of motion is just the boundary term,

$$
\begin{aligned}
\delta I= & \frac{1}{16 \pi} \int_{\partial \mathcal{M}} d^{n} x \sqrt{-h}\left\{\Pi_{\alpha \beta} \delta h^{\alpha \beta}-(1-2 \omega) n^{\mu} F_{\mu \nu} \delta A^{\nu}-n^{\mu} H_{\mu \nu} \delta B^{\nu}\right. \\
& \left.+f^{\prime}\left(B_{\alpha} B^{\alpha}\right)\left(2 B_{\alpha} \delta B^{\alpha}+B_{\alpha} B_{\beta} \delta h^{\alpha \beta}\right)-\frac{1}{2} f\left(B_{\alpha} B^{\alpha}\right) h_{\alpha \beta} \delta h^{\alpha \beta}\right\}
\end{aligned}
$$

where

$$
\Pi_{\alpha \beta}=K_{\alpha \beta}-K h_{\alpha \beta}+(n-1) h_{\alpha \beta} .
$$

Now, if one defines

$$
\begin{aligned}
& S_{\alpha \beta}=\frac{\sqrt{-h}}{16 \pi}\left[\Pi_{\alpha \beta}+\frac{z q}{2}\left(-B_{\gamma} B^{\gamma}\right)^{-1 / 2}\left(B_{\alpha} B_{\beta}-B_{\gamma} B^{\gamma} h_{\alpha \beta}\right)\right], \\
& S_{\alpha}^{L}=-\frac{\sqrt{-h}}{16 \pi}\left[n^{\mu} H_{\mu \alpha}-z q\left(-B_{\gamma} B^{\gamma}\right)^{-1 / 2} B_{\alpha}\right], \\
& S_{\alpha}^{M}=-\frac{\sqrt{-h}}{16 \pi}(1-2 \omega) n^{\mu} F_{\mu \alpha},
\end{aligned}
$$


Then the general variation of the action is ${ }^{1}$

$$
\delta I=\int d^{n} x\left(S_{\alpha \beta} \delta h^{\alpha \beta}+S_{\alpha}^{L} \delta B^{\alpha}+S_{\alpha}^{M} \delta A^{\alpha}\right)
$$

Accordingly we can define a stress tensor complex [20] consisting of the energy density $\mathcal{E}$, energy flux $\mathcal{E}_{i}$, momentum density $\mathcal{P}_{i}$ and spatial stress tensor $\Pi_{i j}$, satisfying the conservation equations

$$
\partial_{t} \mathcal{E}+\partial_{i} \mathcal{E}^{i}=0, \quad \partial_{t} \mathcal{P}_{j}+\partial_{i} \mathcal{P}^{i}{ }_{j}=0
$$

where

$$
\mathcal{E}=2 S_{t}^{t}-S_{L}^{t} B_{t}-S_{M}^{t} A_{t}, \quad \mathcal{E}^{i}=2 S^{i}-S_{L}^{i} B_{t}-S_{M}^{i} A_{t},
$$

and

$$
\mathcal{P}_{i}=-2 S_{i}^{t}+S_{L}^{t} B_{i}+S_{M}^{t} A_{i}, \quad \mathcal{P}_{i}^{j}=-2 S_{i}^{j}+S_{L}^{j} B_{i}+S_{M}^{j} A_{i}
$$

Using the exponential ansatz (9.1) for the metric and gauge potentials,we obtain

$$
\mathcal{E}=\frac{1}{16 \pi}\left[r^{n+z-1} \frac{f}{g}\left[z q^{2}(1-h) j g-2(n-1)(1-g)\right]+(1-2 \omega) Q r^{z} \kappa\right]_{r \rightarrow \infty} .
$$

for the energy density of the black brane.

Employing the large $r$ expansions given in Appendix for the metric functions in the case of $z \neq n-1$ and $z=n-1$ to eq. (10.11), we obtain the rather curious result that

$$
\mathcal{E}=\left\{\begin{array}{cc}
\frac{1}{8 \pi} \frac{(z-1)(n-z-1)}{(n-1)} C_{1}+\frac{(4 \omega-3) Q^{2}}{32 \pi(n-z-1)} r^{z-(n-1)} & z \neq n-1, z \neq 1 \\
\frac{1}{16 \pi(n-1)} C_{1}+\frac{Q^{2}}{32 \pi(n-1)}[(3-4 \omega)(n-1) \ln r+1] & z=n-1
\end{array}\right.
$$

giving a finite energy density if $z<n-1$ regardless of the choice of $\omega$. We remark that for arbitrary $z$ choosing $\omega=\frac{3}{4}$ yields finiteness of energy. However it is straightforward to show that $S_{\alpha}^{M}$ is divergent for large $r$, rendering the variation (10.7) ill-defined, unless $\omega=1 / 2$.

Choosing $\omega=\frac{1}{2}$ gives

$$
\begin{aligned}
I= & \frac{1}{16 \pi} \int_{\mathcal{M}} d^{n+1} x \sqrt{-g}\left(\mathcal{L}_{g}+\mathcal{L}_{m}\right) \\
& +\frac{1}{8 \pi} \int_{\partial \mathcal{M}} d^{n} x z \sqrt{-h}\left(K-(n-1)-\frac{z q}{2} \sqrt{-B_{\alpha} B^{\alpha}}+\frac{1}{2} n^{\mu} F_{\mu \nu} A^{\nu}\right)
\end{aligned}
$$

\footnotetext{
${ }^{1}$ As we shall see, it is only in the canonical ensemble that subsequent equations in this section are defined. Note that $\delta A^{\alpha}=0$ in the grand canonical ensemble.
} 
for the action. Using equations (9.8-9.10) we obtain

$$
C_{1}=\frac{8 \pi(n-1)^{2}}{(z-1)(n+z-1)(n-z-1)}[T S+D \mathcal{Q}] \quad z<n-1,
$$

and applying (10.15) we find

$$
\mathcal{E}=\frac{n-1}{n+z-1}[T S+\mathcal{Q} D] \quad z<n-1,
$$

Since (10.16) is the expression for energy at infinity one may interpret the coefficient conjugate to the electric charge as the chemical potential. Hence

$$
\Phi=D \quad z<n-1
$$

which is consistent with the definition of chemical potential, measured at infinity with respect to the horizon, given as [22]

$$
\begin{aligned}
\Phi & =\left.A_{\mu} \chi^{\mu}\right|_{r \rightarrow \infty}-\left.A_{\mu} \chi^{\mu}\right|_{r=r_{0}}=Q \int^{\infty} \frac{f g}{r^{n-z}} d r+D \\
& =D \quad z<n-1
\end{aligned}
$$

where $\chi^{\mu}=\partial_{t}$ is the null generator of the horizon. For $z=1$ setting $q=0$, substituting (9.13) in eq. (10.11), and expanding the result for large $r$ yields the energy density of Reisner-Nordstrom black brane [23]:

$$
\mathcal{E}=\frac{(n-1)}{16 \pi} m
$$

where $m=\mathcal{C} / n$ is the mass of the AdS black hole. The quantity $\mathcal{C}$ is the conserved charge given by (9.12), which at the horizon in terms of thermodynamic quantities and horizon radius can be expressed as

$$
\mathcal{C}=16 \pi\left[T S+\mathcal{Q} \frac{Q}{(n-2) r_{0}^{n-2}}\right] \quad z=1
$$

and the coefficient of electric charge is exactly AdS chemical potential [23]:

$$
\Phi=D=-Q \int^{r_{0}} \frac{d r}{r^{n-1}}=\frac{Q}{(n-2) r_{0}^{n-2}} \quad z=1
$$

Therefore, the energy density for $z=1$ is

$$
\mathcal{E}=\frac{n-1}{n}(T S+\mathcal{Q} \Phi) .
$$

which, by comparing Eqs. (10.16), (10.17) and (10.22), we see generalizes to

$$
\mathcal{E}=\frac{n-1}{n+z-1}(T S+\mathcal{Q} \Phi),
$$

for asymptotic Lifshitz black branes. 


\section{Concluding Remarks}

Our considerations of Lifshitz gravity coupled to electromagnetism in $(n+1)$ dimensions have indicated a rich array of numerical solutions that depend on the two parameters $h_{0}$ and $Q$. Solutions exist for all topologies, and an extremal limit, denoted by $Q_{c}$ and defined in eq (5.3) exists.

The general effect of $Q$ is to cause metric functions to more slowly approach their asymptotic values, other parameters being equal. We illustrated this with the metric function $f(r)$ : it tends to grow more slowly toward its asymptotic value with increasing charge (as shown in section 6). We also found that increasing charge decreases the boundary length of the Wilson loop (for $n=3$ ) while causing the potential $V$ between two particles to grow more rapidly with increasing string midpoint length $r_{m}$.

We also found that we can extend the thermodynamics of Lifshitz black branes $[15,16,17]$ to the charged case, provided the parameter $z<n-1$. We have obtained an expression, eq. (10.23), for the energy density in terms of the extensive thermodynamic quantities entropy and charge density and their intensive conjugate quantities for asymptotic Lifshitz black branes, generalizing the $z=1$ AdS case (10.22). Extending to values of $z$ larger than this entails a choice of boundary terms that renders the variational principle ill-defined. Commensurate results have been obtained in other models [25] whose solutions smoothly interpolate between Lifshitzlike and AdS-like behaviour as the ratio of $T / \mu$ (with $\mu$ the chemical potential) varies from small to large values.

Further work in this area will involve obtaining a better understanding of how the general effects in this paper affect the dual theories associated with asymptotically Lifshitz spacetimes, and of how quantum-gravitational corrections can likewise modify such effects.

\section{Acknowledgements}

This work was supported in part by the Natural Sciences and Engineering Research Council of Canada.

\section{Appendix}

Here we are willing to explore more details of the eigen modes at large $r$ which have been roughly introduced in eq. (4.4). Here we ignore the universal mode in the set (4.3) by setting $k=0$. The complete solution to (4.3) at large $r$ provided $z \neq n-1$ is

$$
g_{1}(r)=-\frac{C_{1} G_{1}}{r^{z+n-1}}-\frac{C_{2} G_{2}}{r^{(z+n-1+\sqrt{\gamma}) / 2}}-\frac{C_{3} G_{3}}{r^{(z+n-1-\sqrt{\gamma}) / 2}}-\frac{(n-2 z) Q^{2}}{\left.4(n+z-2)(n-z-1)^{2} r^{2 n-2},\right)}
$$




$$
\begin{aligned}
& h_{1}(r)=-\frac{C_{1}}{r^{z+n-1}}-\frac{C_{2}}{r^{(z+n-1+\sqrt{\gamma}) / 2}}-\frac{C_{3}}{r^{(z+n-1-\sqrt{\gamma}) / 2}}+\frac{(2 n-z-2) Q^{2}}{\left.4(n+z-2)(n-z-1)^{2} r^{2 n-2} \cdot 2\right)} \\
& j_{1}(r)=-\frac{C_{1} J_{1}}{r^{z+n-1}}-\frac{C_{2} J_{2}}{r^{(z+n-1+\sqrt{\gamma}) / 2}}-\frac{C_{3} J_{3}}{r^{(z+n-1-\sqrt{\gamma}) / 2}}-\frac{Q^{2}}{4(n-z-1)^{2} r^{2 n-2}},
\end{aligned}
$$

where

$$
\begin{aligned}
& \gamma=9 z^{2}-2(3 n+1) z+\left(n^{2}+6 n-7\right) \\
& G_{1}=\frac{z(z-1)}{(n-1)^{2}}, \quad G_{2}=\frac{z-1}{n-1}, \quad G_{3}=\frac{z-1}{n-1} \\
& J_{1}=-\frac{z(z-2+n)}{(n-1)^{2}}, \quad J_{2}=\frac{n-3 z+1-\sqrt{\gamma}}{2(n-1)}, \quad J_{3}=\frac{n-3 z+1+\sqrt{\gamma}}{2(n-1)}
\end{aligned}
$$

If one inserts the above expression in equation for the small perturbation of $f(r)$, i.e

$r \frac{d}{d r} f_{1}(r)=(n-2+2 z) g_{1}(r)-\frac{z(z-1)}{n-1} h_{1}(r)+(z-1) j_{1}(r)-\frac{Q^{2}}{4(n-1) r^{2 n-2}}+\frac{(n-2) k}{2 r^{2}}$,

then again the universal $1 / r^{2}$ mode decays away at infinity and one obtains

$$
f_{1}(r)=\frac{C_{1} F_{1}}{r^{z+n-1}}+\frac{C_{2} F_{2}}{r^{(z+n-1+\sqrt{\gamma}) / 2}}+\frac{C_{3} F_{3}}{r^{(z+n-1-\sqrt{\gamma}) / 2}}+\frac{Q^{2}}{4(n-z-1)(n-1) r^{2 n-2}},
$$

with the constraint that $f_{1}(r)$ should goes to zero as $r$ goes to infinity and

$$
\begin{aligned}
& F_{1}=\frac{z(z-1)(n-z-1)}{(n-1)^{2}(n+z-1)}, \quad F_{2}=\frac{z(n+1)-n^{2}-2 z^{2}+1+(n-1) \sqrt{\gamma}}{2(n-1)(n-z-1)}, \\
& F_{3}=\frac{z(n+1)-n^{2}-2 z^{2}+1-(n-1) \sqrt{\gamma}}{2(n-1)(n-z-1)} .
\end{aligned}
$$

For $z=n-1$ solutions are given by:

$$
\begin{aligned}
g_{1}(r) & =-\frac{(n-2)}{2(n-1)^{2}} \frac{\left(C_{1} \ln r+C_{2}\right)}{r^{2 n-2}}-\frac{(3 n-4)}{4(n-1)^{3}} \frac{C_{1}}{r^{2 n-2}} \\
& +\left[(n-2)(\ln r)^{2}+\frac{(3 n-4) \ln r}{n-1}+\frac{5 n-6}{2(n-1)^{2}}\right] \frac{Q^{2}}{8(n-1) r^{2 n-2}}, \\
h_{1}(r) & \left.=-\frac{\left(C_{1} \ln r+C_{2}\right)}{2(n-1) r^{2 n-2}}-\frac{C_{1}}{4(n-1)^{2} r^{2 n-2}}+\left[(\ln r)^{2}+\frac{\ln r}{n-1}+\frac{1}{2(n-1)^{2}}\right] \frac{(11.10)}{8 r^{2 n-2}}\right) \\
j_{1}(r) & =\frac{(2 n-3)}{2(n-1)^{2}} \frac{\left(C_{1} \ln r+C_{2}\right)}{r^{2 n-2}}+\frac{(2 n-3)}{4(n-1)^{3}} \frac{C_{1}}{r^{2 n-2}} \\
& -\left[(2 n-3)(\ln r)^{2}+\frac{(2 n-3) \ln r}{n-1}+\frac{4 n-5}{2(n-1)^{2}}\right] \frac{Q^{2}}{8(n-1) r^{2 n-2}}, \\
f_{1}(r) & =\frac{(3 n-4)}{4(n-1)^{3}} \frac{C_{1}}{r^{2 n-2}}-[(n-1) \ln r+1] \frac{(3 n-4) Q^{2}}{8(n-1)^{3} r^{2 n-2}} .
\end{aligned}
$$

which are relevant to asymptotic Lifshitz background. 


\section{References}

[1] J. M. Maldacena, The large $N$ limit of superconformal field theories and supergravity Adv. Theor. Math. Phys. 2, 231 (1998)[Int. J. Theor. Phys. 38, 1113 (1999)].

[2] P. Koroteev and M. Libanov, On Existence of Self-Tuning Solutions in Static Braneworlds without Singularities, JHEP 0802 (2008) 104 [arXiv:0712.1136].

[3] S. Kachru, X. Liu and M. Mulligan, Gravity Duals of Lifshitz-like Fixed Points Phys. Rev. D 78106005 (2008).

[4] S. A. Hartnoll, Lectures on holgraphic methods for condensed matter physics JHEP [e-Print: arXiv:0903.3246].

[5] D. S. Rokhsar and S. A. Kivelson, Superconductivity and the Quantum Hard-Core Dimer Gas Phys. Rev. Lett. 612376 (1988).

[6] E. Ardonne, P. Fendley and E. Frandkin, Topological order and conformal quantum critical points Annal Phys. 310493 (2004).

[7] A. Vishwanath, L. Balents, and T. Senthil, Quantum Criticality and Deconfinement in Phase Transitions Between Valence Bond Solids Phys. Rev. B 69224416 (2004).

[8] U. H. Danielsson and L. Thorlacius Black holes in asymptotically lifshitz spacetime JHEP 0903070 (2009).

[9] R. B. Mann, Lifshitz Topological Black Holes, JHEP 0906 (2009) 075.

[10] E. J. Brynjolfsson, U. H. Danielsson, L. Thorlacius and T. Zingg Holographic Superconductors with Lifshitz Scaling J. Phys. A43 065401 (2010).

[11] E. J. Brynjolfsson, U. H. Danielsson, L. Thorlacius and T. Zingg Holographic Models with Anisotropic Scaling, arXiv:1004.5566[hep-th]

[12] E. J. Brynjolfsson, U. H. Danielsson, L. Thorlacius and T. Zingg Black Hole Thermodynamics and Heavy Fermion Metals JHEP 08027 (2010).

[13] E. J. Brynjolfsson, U. H. Danielsson, L. Thorlacius and T. Zingg [e-Print: arXiv:1004.5566]

[14] D. Pang On Charged Lifshitz Black Holes [e-Print: arXiv:0911.2777v2]

[15] G. Bertoldi, B. A. Burrington and A. Peet, Black holes in asymptotically Lifshitz spacetimes with arbitrary critical exponent, Phys. Rev. D 80126003 (2009) [arXiv:0905.3183].

[16] G. Bertoldi, B. A. Burrington and A. W. Peet, Thermodynamics of black branes in asymptotically Lifshitz spacetimes Phys. Rev. D 80126004 (2009) [arXiv:0907.4755]

[17] M. H. Dehghani and R. B. Mann, Thermodynamics of Lovelock-Lifshitz Black Branes, Phys. Rev. D 82, 064019 (2010) [arXiv: 1006.3510] 
[18] D. G. Boulware and S. Deser, String-generated gravity models, Phys. Rev. Lett. 55, 2656 (1985).

[19] J. M. Maldacena Wilson loops in large N Field theories Phys. Rev. Lett. 804859 (1998); S. J. Rey and J. T. Yee, Macroscopic strings as heavy quarks in large $N$ gauge theory and anti-de Sitter supergravity Eur. Phys. J. C22 379 (2001).

[20] S. F. Ross and O. Saremi, Holographic stress tensor for non-relativistic theories, JHEP 0909 (2009) 009.

[21] K. Copsey and R. B. Mann Pathologies in Asymptotically Lifshitz Spacetimes arXiv: 1011.3502 .

[22] M. M. Caldarelli, G. Cognola, and D. Klemm, Class. Quantum Grav. 17, 339 (2000).

[23] M. H. Dehgahni and A. Khodam-Mohammadi, Phys. Rev. D 67, 084006 (2003).

[24] R. B. Mann and S. F. Ross Cosmological production of charged black hole pairs, Phys. Rev. D 52, 2254 (1995).

[25] G. Bertoldi, B. A. Burrington and A. Peet, Thermal behavior of charged dilatonic black branes in AdS and UV completions of Lifshitz-like geometries Phys. Rev. D 82 106013 (2010) [arXiv:1007.1464];G. Bertoldi, B. A. Burrington, A. W. Peet, I. G. Zadeh, Lifshitz-like black brane thermodynamics in higher dimensions [arXiv:1101.1980]. 